\title{
DIABETES TIPO 1 E INSULINORRESISTENCIA: TASA ESTIMADA DE DISPOSICIÓN DE LA GLUCOSA Y SU CORRELACIÓN CON COMPLICACIONES CRÓNICAS
}

\author{
TYPE 1 DIABETES AND INSULIN RESISTANCE: ESTIMATED \\ GLUCOSE DISPOSAL RATE AND ITS CORRELATION WITH \\ CHRONIC COMPLICATIONS
}

Gabriela Caeiro', Silvana A Romero González¹, Jimena Itria', Jorge Waitman²

\begin{abstract}
RESUMEN
Introducción: la insulinorresistencia (IR) en diabetes tipo 1 (DM1) se asocia a mayor riesgo de complicaciones. El gold standard para evaluar insulinosensibilidad (IS) es el clamp euglucémico-hiperinsulinémico, aunque no se utiliza en la práctica clínica. La tasa estimada de disposición de la glucosa (TeDG) ha sido validada para estimar IS en DM1.

Objetivos: relacionar IR en DM1 (TeDG) con complicaciones macro y microvasculares en pacientes asistidos en un Servicio de Diabetología.

Materiales y métodos: se realizó un estudio transversal. La TeDG fue calculada utilizando $\mathrm{HbA} 1 \mathrm{c}$, perímetro de cintura y presencia de hipertensión.

Resultados: se incluyeron 446 pacientes, 57\% hombres, edad promedio 38 años y 15 años de evolución de la enfermedad. Los pacientes en el tercil más bajo de TeDG (>IR) tenían mayor edad, tiempo de evolución, IMC, dosis de insulina, mayores niveles de LDL y TGC y peor control metabólico. Eran más sedentarios y tenían mayor indicación de estatinas, aspirina, IECA/ARAll y metformina. Además registraban mayor prevalencia de complicaciones microvasculares y enfermedad cardiovascular $(p<0,05)$. En el análisis multivariado, estos pacientes tuvieron mayor riesgo de nefropatía (OR 3,37 [95\% $\mathrm{Cl} 1,92-5,90]$ ) y de cualquier complicación de la diabetes (OR 2,44 [95\% Cl 1,41-4,24]).

Conclusiones: una menor TeDG se asoció a mayor presencia de complicaciones y comorbilidades. Esta herramienta podría resultar útil para identificar pacientes con mayor riesgo de complicaciones a fin de implementar estrategias más agresivas para el control de los factores de riesgo.
\end{abstract}

Palabras clave: diabetes mellitus tipo 1, resistencia a la insulina, complicaciones de la diabetes.

Revista de la Sociedad Argentina de Diabetes 2017; Vol. 51 (38-46)

\begin{abstract}
Introduction: insulin resistance (IR) in type 1 diabetes (T1D) is associated with a higher risk of complications in these patients. The gold standard for measuring insulin sensitivity is the hyperinsulinemic-euglycemic clamp although not used in clinical practice. The estimated glucose disposal rate (eGDR) has been validated to estimate insulin sensitivity.

Objectives: the objective was to correlate IR in T1D (eGDR), with macro and microvascular complications in patients assisted in a Diabetology Service.

Materials and methods: we performed a cross-sectional study. The eGDR was calculated using HbA1c, waist circumference, and hypertensive status.

Results: 446 patients with prior clinical diagnosis of T1D were included. A 57\% of patients were male; mean age 38 years and an average of 15-year evolution of diabetes. The patients in the lowest eGDR tertile (>IR) were older, with longer time of evolution, larger BMI, higher insulin doses, higher levels of triglycerides and $L D L$, and poorer metabolic control. They were more sedentary and had more use of statins, aspirin, ACE inhibitor or ARBs, and metformin. They showed higher prevalence of microvascular complications and higher cardiovascular disease $(p<0.05)$. In the multivariate analysis, the lowest tertile patients had the highest risk of nephropathy (OR 3.37 [95\% Cl 1.92-5.90]) and any complication of diabetes (OR 2.44 [95\% Cl 1.41-4.24]). Conclusions: a lower eGDR was associated with higher presence of complications and comorbidities. This clinical tool could become useful to identify patients with higher risk of complications, in order to implement more aggressive strategies to control risk factors.
\end{abstract}

Key words: type 1 diabetes mellitus, insulin resistance, diabetes complications.

Revista de la Sociedad Argentina de Diabetes 2017; Vol. 51 (38-46)
Médica del Servicio de Diabetes, Hospital Córdoba, Córdoba, Argentina

2 Jefe del Servicio de Diabetes, Hospital Córdoba; Director de la Carrera de Posgrado de Especialistas en Diabetología (Universidad Nacional de Córdoba, UNC); ex Profesor de la Cátedra I de Semiología, Hospital Córdoba (UNC), Córdoba, Argentina
Contacto de la autora: Gabriela Caeiro

E-mail: caeirogabriela@hotmail.com

Correspondencia: Av. Patria 656 (CP 5000), Córdoba, Argentina Te: (0351) 434-9090

Fecha de trabajo recibido: 01/06/17

Fecha de trabajo aceptado: 31/06/17

Conflictos de intereses: los autores declaran que no existe conflicto de interés 


\section{INTRODUCCIÓN}

El mecanismo fisiopatológico principal en la diabetes tipo 1 (DM1) es la insulinodeficiencia relacionada con la destrucción inmune de las células beta del páncreas. Sin embargo, el mantenimiento de la normoglucemia en estos pacientes es en realidad resultado de la interacción entre la secreción de las células $\beta$ y la sensibilidad a la insulina en el hígado y en los tejidos periféricos. En el año 1977 el Dr. Henry Ginsberg demostró que los cambios en la sensibilidad a la insulina juegan un papel en la DM11. Desde entonces varios estudios confirmaron la presencia de insulinorresistencia en estos pacientes ${ }^{2-4}$.

La insulinorresistencia (IR) en DM1 se asocia a un mayor riesgo cardiovascular y presencia de complicaciones microvasculares, principalmente nefropatía diabética ${ }^{5-6}$. En poblaciones con altos índices de diabetes tipo 2 y obesidad, las personas con DM1 pueden compartir los factores genéticos y ambientales que resultan en una reducción de la sensibilidad a la insulina, un fenómeno conocido como "diabetes doble"7-8.

El gold standard para la medición de insulinosensibilidad es el clamp euglucémico-hiperinsulinémico. Esta técnica no resulta práctica para su aplicación en el ámbito clínico dado que es costosa y técnicamente engorrosa.

La tasa estimada de disposición de la glucosa oTeDG es una fórmula que puede calcularse utilizando mediciones clínicas (hemoglobina glicosilada, presencia de hipertensión y perímetro de cintura); muestra una buena correlación con la IR medida por el clamp euglucémico-hiperinsulinémico y ha sido validada para la estimación de insulinosensibilidad en DM19-11.

La población argentina incluye etnias de alto riesgo para el desarrollo de diabetes tipo 2. Además la prevalencia de obesidad y sobrepeso se encuentra en franco aumento; actualmente se estima que la de la obesidad es del $20,8 \%$ y la del sobrepeso del $37,1 \%{ }^{12}$. Estas condiciones podrían determinar una mayor presencia de IR en pacientes con DM1 en nuestro medio. En base a esta hipótesis, se realizó un estudio transversal para evaluar la presencia de insulinorresistencia medida a través de la TeDG en nuestra población de pacientes con DM1 y su asociación con complicaciones crónicas y factores de riesgo cardiovascular.

\section{MATERIALES Y MÉTODOS}

Se realizó un estudio descriptivo, observacional y transversal en el Servicio de Diabetología del Hospital Córdoba, Provincia de Córdoba, Argentina. Se incluyeron pacientes con diagnóstico clínico de DM1 realizado por un médico especialista en Diabetología. Se valoraron características clínicas adicionales como el uso de insulina dentro del primer año de diagnóstico, edad menor de 30 años al diagnóstico, hospitalización por cetoacidosis diabética y presencia de anticuerpos anti-GAD (anticuerpos antiglutamato decarboxilasa). Se excluyeron pacientes con diabetes tipo 2 , embarazadas y menores de 15 años.

Los datos se recolectaron a partir de la revisión de historias clínicas. Se obtuvo información de las siguientes variables: edad, sexo, tiempo de evolución de la enfermedad, tratamiento, dosis de insulina (UI/Kg/día), peso, talla, IMC, presencia de hipertensión arterial, dislipemia, complicaciones crónicas microvasculares (retinopatía, nefropatía, neuropatía periférica), complicaciones macrovasculares (accidente cerebrovascular, infarto agudo de miocardio y enfermedad vascular periférica), tabaquismo, sedentarismo, antecedente heredofamiliar de diabetes tipo 2, HbA1c (medida por inmunoturbidimetría o HPLC), creatininemia, perfil lipídico, excreción urinaria de albúmina y clearence de creatinina calculado por la fórmula modification of diet in renal disease study (MDRD) ${ }^{13}$

Para evaluar la insulinosensibilidad se utilizó la fórmula de la tasa estimada de disposición de la glucosa (TeDG) que se calculó al utilizar el valor de $\mathrm{HbA} 1 \mathrm{c}$, perímetro de cintura $(\mathrm{PC})$ y presencia de hipertensión arterial (HTA)

TeDG $(\mathrm{mg} / \mathrm{kg} / \mathrm{min})=21.158+(-0,09 \times P C)+$ $(-3,407 \times$ HTA $)+(-0,551 \times$ HbA1c) 
La presencia de hipertensión se definió como el antecedente de HTA o el uso de medicamentos antihipertensivos $(0=\mathrm{No}, 1=\mathrm{Sí})$, el perímetro de cintura medido en centímetros y la HbA1c expresada en porcentaje (\%).

Se determinó dislipemia como la presencia de una o más de las siguientes alteraciones en el perfil lipídico: LDL-c $\geq 100 \mathrm{mg} / \mathrm{dl}$, triglicéridos $\geq 150 \mathrm{mg} / \mathrm{dl}$, HDL-c $<50 \mathrm{mg} / \mathrm{dl}$ en mujeres ó $<40 \mathrm{mg} / \mathrm{dl}$ en hombres y/o tratamiento hipolipemiante ${ }^{14}$.

Se definió sedentarismo como la falta de cumplimiento de los niveles recomendados de actividad física (150 minutos o más de actividad física de intensidad moderada a vigorosa por semana, distribuida en al menos tres días por semana) ${ }^{15}$.

Para describir la obesidad abdominal se utilizó la definición de la Federación Internacional de Diabetes (IDF): perímetro de cintura en hombres $\geq 94 \mathrm{~cm}$ y en mujeres $\geq 80 \mathrm{~cm}^{16}$.

La presencia de retinopatía diabética (RD) se evaluó a través del fondo de ojo realizado por un médico oftalmólogo. Los hallazgos se clasificaron como: normal, retinopatía diabética no proliferativa (RPDNP) y retinopatía diabética proliferativa (RPDP) ${ }^{17}$. Para el análisis multivariado, la variable se analizó como presencia o ausencia de retinopatía diabética.

La nefropatía diabética se evaluó según la excreción urinaria de albúmina (EUA) medida en muestras aisladas de orina o en recolecciones de 24 hs. Una EUA $<20 \mu \mathrm{gr} / \mathrm{min}$ ó <30 mg/día se clasificó como normoalbuminuria, entre 20-200 $\mu \mathrm{gr} / \mathrm{min}$ ó 30-300 mg/día como microalbuminuria y valores $>200 \mu \mathrm{gr} / \mathrm{min}$ ó >300 mg/día como proteinuria franca. Para el diagnóstico de nefropatía diabética se consideraron al menos dos mediciones de EUA mayores a $20 \mu \mathrm{gr} / \mathrm{min}$ ó $30 \mathrm{mg} / \mathrm{día}^{18}$. Se definió enfermedad renal crónica al estadio 3 ó más estimado por el cálculo de tasa de filtrado glomerular por MDRD. Para el análisis multivariado se consideró la presencia o ausencia de nefropatía diabética y enfermedad renal crónica.

El diagnóstico de neuropatía diabética pe- riférica se estableció con la valoración de los síntomas y signos mediante la escala de síntomas de neuropatía (Neuropathy Symptom Scale, NSS) y la escala de alteraciones neurológicas (Neuropathy Disability Score, NDS) ${ }^{19}$, donde se valora la sensibilidad vibratoria, táctil, termoalgésica, fuerza muscular y reflejos osteotendinosos. Esta última tiene un puntaje máximo de 12 puntos (seis por cada miembro inferior) y resulta positiva para neuropatía con un valor igual o mayor a 3 puntos. Para el análisis multivariado, la variable se analizó como presencia o ausencia de neuropatía.

Se consideró como presencia de complicaciones macrovasculares el antecedentes de IAM, bypass aortocoronario, angioplastia percutánea, test de stress positivo, historia de ACV o enfermedad vascular periférica $(A B \mid<0,9$, angiografía o historia de revascularización).

La historia familiar de diabetes tipo 2 se definió como la existencia de familiares de primer grado con diagnóstico de diabetes con inicio después de los 30 años y uso de antidiabéticos orales en algún momento de la enfermedad.

La TeDG se dividió en terciles (tercil 1: $<6,54$; tercil 2: 6,54 a 8,53 y tercil 3: $>8,53$ ), siendo el tercil menor el que representa a aquellos pacientes con mayor insulinorresistencia (IR) y los más altos aquellos con menor IR (mayor insulinosensibilidad [IS]).

Se estimó la prevalencia de complicaciones y comorbilidades por terciles de TeDG y se realizó un análisis multivariado para definir su asociación con la insulinorresistencia.

Luego de completar el formulario de datos a partir del estudio de las historias clínicas, se efectuó un análisis estadístico descriptivo según la naturaleza de las variables; para las mensurables se utilizaron medidas de estadística central y de dispersión (media, error standard, desvío standard), y para las de tipo categórico se estudiaron las frecuencias absolutas y relativas porcentuales. Para la ejecución del análisis inferencial se empleó el test de Chi cuadrado y test de diferencias de medias. Se fijó como nivel de significación 
$\alpha=0,05$. Todos los análisis se realizaron con el Software Stata 14.

El proyecto fue aprobado por el Comité de Capacitación y Docencia del Hospital Córdoba.

\section{RESULTADOS}

Se incluyeron 446 pacientes con diagnóstico clínico de DM1. El 72,6\% tenía menos de 30 años de edad al diagnóstico de su enfermedad, el 35,8\% presentó hospitalización por cetoacidosis diabética, el 96\% requirió insulina antes del primer año de diagnóstico y sólo el 1\% tenía medición de anticuerpos anti-GAD (este estudio no se encuentra disponible en nuestro medio). El 57,4\% de la población estaba formada por hombres, con una edad media de $38 \pm 15$ años y un promedio de 15 años de evolución de la enfermedad. El $10 \%$ se encontraba en tratamiento combinado con metformina. El promedio de IMC fue de $25,28 \pm 4,6$ y el promedio de TeDG de 7,31.

Con respecto a la presencia de factores de riesgo asociados, un 22,6\% tenía hipertensión arterial, 28\% dislipemia, 30\% era tabaquista, 38\% era sedentario, 25\% tenía sobrepeso y $15 \%$ obesidad.

En relación al perímetro de cintura, presentaron obesidad abdominal el 38,9\% de las mujeres y el $23,4 \%$ de los hombres. De la totalidad de pacientes analizados, más de la mitad $(53,8 \%)$ tenía antecedentes heredofamiliares de DM2.

Un 55,6\% de los pacientes manifestó alguna complicación crónica de la diabetes: el $32 \%$ tenía retinopatía, el 32,5\% nefropatía, el $29,3 \%$ neuropatía periférica y el 10,3\% complicaciones macrovasculares.

En el análisis univariado se encontró que los pacientes en el tercil más bajo de TeDG (mayor IR) comparados con aquellos con tercil más alto (mayor IS) tenían mayor edad (45 años vs 33 años, $p=0,00001$ ), mayor tiempo de evolución de la enfermedad (18 años vs 13 años, $p=0,00001)$, mayor IMC (28 vs 23, $\mathrm{p}=0,00001)$, mayor dosis de insulina/ $\mathrm{kg}$ de peso $(1,34 \mathrm{U} / \mathrm{Kg} / \mathrm{d}$ vs 0,79 U/Kg/d, $p<0,0001)$, mayor nivel de triglicéridos $(159 \mathrm{mg} / \mathrm{dl}$ vs $102 \mathrm{mg} / \mathrm{dl}, \mathrm{p}=0,00001)$ y LDL (115 mg/dl vs $104 \mathrm{mg} / \mathrm{dl}, p=0,0039)$, menores niveles de HDL (48 mg/dl vs $51 \mathrm{mg} / \mathrm{dl}, \mathrm{p}=0,0005)$ y peor control metabólico (HbA1c 10\% vs 9,2\%, $\mathrm{p}=0,001)$. Además eran más sedentarios (52\% vs $31 \%$ ) y tenían mayor indicación de estatinas (47\% vs $14 \%$ ), aspirina (29\% vs $4 \%)$, IECA o ARAII (64\% vs 18\%) y metformina $(19 \%$ vs $3 \%$, p <0,0001). Registraban mayor prevalencia de retinopatía (45\% vs $24 \%$ ), nefropatía (48\% vs 19\%), neuropatía periférica $(44 \%$ vs $19 \%)$ y mayor enfermedad cardiovascular (21\% vs $5 \%)(p<0,001)$ (Tabla 1$)$.

En el análisis multivariado (ajustado por edad, sexo, duración de diabetes, dislipemia, tabaquismo y sedentarismo) los pacientes del tercil 1 vs tercil 3 tuvieron mayor riesgo de nefropatía (OR 3,37 [95\% Cl 1,92-5,90] $p=0,0001$ ) y mayor riesgo de cualquier complicación asociada a la diabetes (OR 2,44 [95\% Cl 1,41-4,24] $p=0,001)$. No hubo relación significativa con retinopatía diabética (OR 1,46 $[95 \% \mathrm{Cl} 0,84-2,54] \mathrm{p}=0,17)$, neuropatía periférica (OR 1,46 [95\% Cl 0,80-2,65] $p=0,21$ ) y enfermedad cardiovascular (OR 2,21 [95\% Cl $0,89-5,48] \mathrm{p}=0,085$ ) (Figura 1).

Además se realizó un análisis comparativo del tercil 2 vs tercil 3 (ajustado por edad, sexo, duración de la diabetes, dislipemia, sedentarismo y tabaquismo) que demostró -de manera similar a la comparación del tercil 1 vs tercil 3- mayor riesgo para nefropatía (OR $1,83[\mathrm{Cl} 95 \% 1,05-3,16 \mathrm{p}=0,031])$ y cualquier complicación asociada a la diabetes (OR 1,71 [Cl 95\% 1,03-2,83 p=0,037]) (Figura 2). 


\begin{tabular}{|c|c|c|c|c|c|}
\hline Variables & $\begin{array}{l}\text { Total de } \\
\text { pacientes }\end{array}$ & $\begin{array}{c}\text { Tercil } 1 \\
(\mathrm{TeDG}<6,54)\end{array}$ & $\begin{array}{c}\text { Tercil } 2 \\
\text { (TeDG 6,54-8,53) }\end{array}$ & $\begin{array}{c}\text { Tercil } 3 \\
(\text { TeDG }>\mathbf{8 , 5 3})\end{array}$ & $\mathbf{p}$ \\
\hline Edad & $37,9 \pm 14,2$ & $44,9 \pm 14,3$ & $35,7 \pm 12,7$ & $33 \pm 12,7$ & 0,00001 \\
\hline Sexo masculino (\%) & 57 & 56,38 & 63,76 & 52,03 & 0,37 \\
\hline IMC & $25,28 \pm 4,6$ & $27,94 \pm 5,17$ & $24,75 \pm 4$ & $23,14 \pm 3,31$ & 0,00001 \\
\hline $\begin{array}{l}\text { Duración de la } \\
\text { diabetes }\end{array}$ & $15,58 \pm 9,8$ & $18,19 \pm 11,3$ & $15,3 \pm 8,63$ & $13,23 \pm 8,6$ & 0,0001 \\
\hline PC & $87,8 \pm 13,86$ & $98,71 \pm 13,78$ & $88,76 \pm 10,4$ & $78,01 \pm 8,15$ & 0,00001 \\
\hline TeDG & $7,31 \pm 2,43$ & $4,47 \pm 1,57$ & $7,73 \pm 0,57$ & $9,75 \pm 0,82$ & 0,00001 \\
\hline $\mathrm{HbA1c}$ & $9,81 \pm 2,5$ & $10,3 \pm 2,78$ & $9,84 \pm 2,23$ & $9,26 \pm 2,37$ & 0,0001 \\
\hline $\mathrm{HDL}$ & $49,8 \pm 14,16$ & $48,03 \pm 14,6$ & $50,19 \pm 14,06$ & $51,37 \pm 13,69$ & 0,0005 \\
\hline LDL & $109 \pm 38,6$ & $115,47 \pm 43,6$ & $107,91 \pm 37,52$ & $103,7 \pm 3,54$ & 0,0039 \\
\hline Triglicéridos & $132,6 \pm 102,4$ & $158,83 \pm 125,92$ & $136,24 \pm 105,49$ & $102,58 \pm 54,83$ & 0,00001 \\
\hline HTA (\%) & 22,65 & 63,8 & 4 & 0 & $<0,0001$ \\
\hline Sedentarismo (\%) & 38,34 & 51,8 & 31,5 & 31,8 & $<0,0001$ \\
\hline Estatinas (\%) & 28 & 46,98 & 22,8 & 14,19 & $<0,0001$ \\
\hline AAS (\%) & 15,25 & 28,86 & 12,75 & 4 & $<0,0001$ \\
\hline IECA/ARA (\%) & 36,1 & 64,43 & 25,5 & 18,24 & $<0,0001$ \\
\hline Metformina (\%) & 10,31 & 19,46 & 8,72 & 2,7 & $<0,0001$ \\
\hline $\begin{array}{l}\text { Dosis de insulina } \\
\text { (UI/Kg/día) }\end{array}$ & $1,28 \pm 0,28$ & $1,34 \pm 0,32$ & $0,84 \pm 0,25$ & $0,79 \pm 0,24$ & $<0,0001$ \\
\hline $\begin{array}{l}\text { Retinopatía } \\
\text { diabética (\%) } \\
\text {. No proliferativa } \\
\text {. Proliferativa }\end{array}$ & $\begin{array}{r}32 \\
14,3 \\
17,7\end{array}$ & $\begin{array}{r}44,97 \\
16,1 \\
29,5\end{array}$ & $\begin{array}{r}26,85 \\
17,4 \\
9,4\end{array}$ & $\begin{array}{l}24,3 \\
9,46 \\
14,9\end{array}$ & 0,0001 \\
\hline $\begin{array}{l}\text { Nefropatía } \\
\text { diabética (\%) }\end{array}$ & 32,4 & 47,65 & 30,87 & 18,92 & 0,0001 \\
\hline $\begin{array}{l}\text { Neuropatía } \\
\text { periférica (\%) }\end{array}$ & 29,37 & 44,3 & 24,16 & 19,59 & 0,0001 \\
\hline $\begin{array}{l}\text { Complicaciones } \\
\text { macrovasculares }(\%)\end{array}$ & 10,3 & 21,48 & 4,03 & 5,41 & 0,001 \\
\hline $\begin{array}{l}\text { Todas las } \\
\text { complicaciones (\%) }\end{array}$ & 55,6 & 74,5 & 54,36 & 37,84 & 0,0001 \\
\hline $\begin{array}{l}\text { Antecedentes } \\
\text { heredofamiliares } \\
\text { DM2 (\%) }\end{array}$ & 55,6 & 63,76 & 53,02 & 44,59 & 0,004 \\
\hline
\end{tabular}

PC: perímetro de cintura; HTA: hipertensión arterial; AAS: ácido salicílico.

Tabla 1: Datos demográficos y características clínicas de la población de acuerdo a terciles deTeDG. 


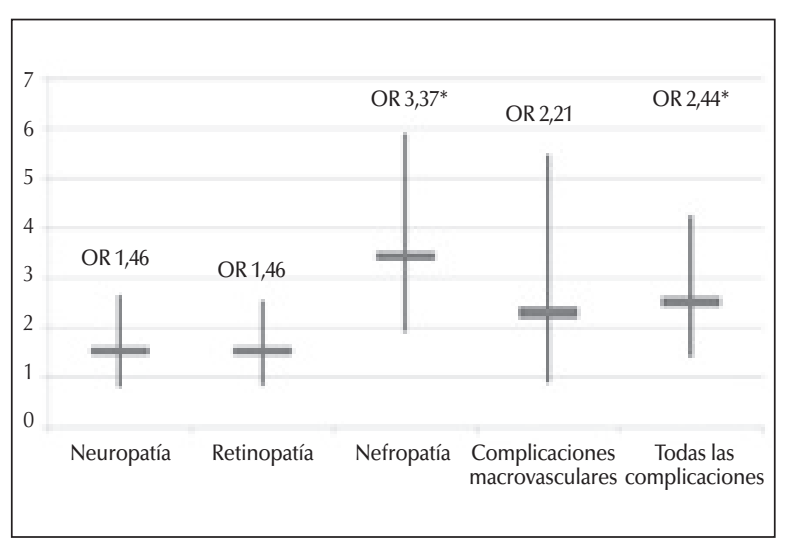

${ }^{*} p<0,05$

Figura 1: OR de complicaciones macro y microvasculares comparando tercil 1 vs 3 .

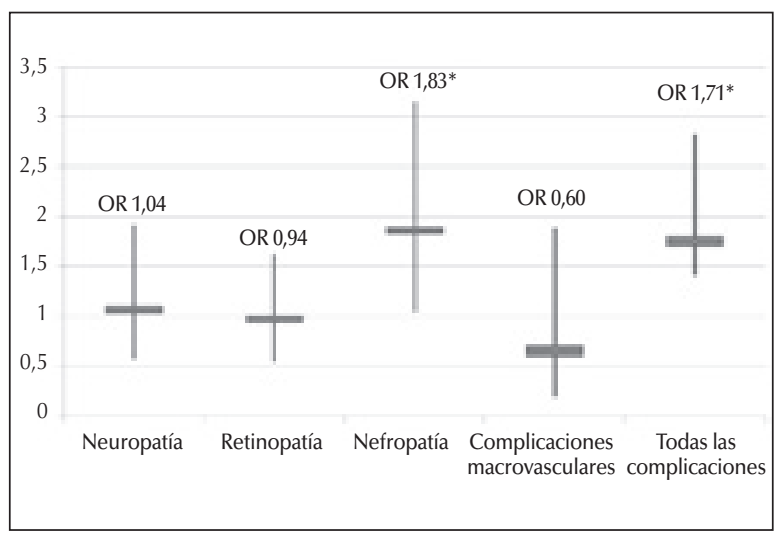

${ }^{*} p<0,05$.

Figura 2: OR de complicaciones macro y microvasculares comparando tercil 2 vs 3.

\section{DISCUSIÓN}

Durante las últimas dos décadas hubo un aumento en la prevalencia del sobrepeso en DM1 y estas cifras se han triplicado desde 1980 hasta $1990^{20}$, siguiendo la tendencia de la población general ${ }^{21}$. Se estima que la prevalencia de sobrepeso y obesidad en adultos jóvenes con DM1 es de aproximadamente un $50 \%{ }^{22-24}$, y entre el 8 y el $40 \%$ presentan síndrome metabólico, lo que sugiere la aparición de un nuevo fenotipo en estos pacientes. Se considera que varios factores pueden determinar esta situación: la insulinoterapia intensificada, el consumo de alimentos de alta densidad calórica, el sedentarismo y principalmente los antecedentes genéticos ${ }^{25}$. En nuestro estudio encontramos que el $25 \%$ de los pacientes tenía sobrepeso, 15\% obesidad, $13 \%$ bajo peso y el $47 \%$ tenía IMC normal coincidiendo con los datos publicados por otros autores ${ }^{26}$. El cambio en el tratamiento en las últimas décadas con un régimen de insulina intensificado podría contribuir al aumento de peso ${ }^{27}$. Los pacientes incluidos en el tercil inferior en nuestro estudio utilizaban una mayor dosis de insulina por kg de peso en comparación al tercil superior.

La obesidad no es el único factor de riesgo para el desarrollo de insulinorresistencia en DM1. En nuestro trabajo los pacientes con IR presentaron una alta prevalencia de antecedentes heredofamiliares de DM2 (más del $50 \%$ ). Dado que la carga genética en el síndrome metabólico es alta, podría ser posible que las personas con diabetes doble hereden los genes de DM2 de sus padres. Estos hallazgos concuerdan con los datos del estudio de Thorn et al., quienes encontraron que los pacientes con antecedentes familiares de DM2 tuvieron una aparición más tardía de la DM1, mayor IMC, mayor circunferencia de cintura, triglicéridos más altos y concentraciones mayores de $\mathrm{A} 1 \mathrm{C}$, mientras que no se observó diferencia en la presión arterial ni en la prevalencia de complicaciones de la diabetes ${ }^{28}$.

El papel de la IR en el desarrollo y progresión de las complicaciones macro y microvasculares en pacientes con DM1 es cada vez más reconocido. En este estudio encontramos que los pacientes con niveles más bajos de TeDG tienen un mayor riesgo de complicaciones micro y macrovasculares aunque luego de ajustar por edad, sexo, duración de diabetes, dislipemia, tabaquismo y sedentarismo sólo se asoció con mayor prevalencia de nefropatía y el compuesto de cualquier complicación asociada a la diabetes. En 1968, Martin y Stocks demostraron que la IR se asoció a complicaciones microvasculares ${ }^{29}$. Chillarón et al. evaluaron pacientes con DM1 
en España y encontraron que aquellos con valores más bajos de TeDG se asociaron de manera significativa con complicaciones microvasculares $^{30}$. Sin embargo no mostró asociación con eventos cardiovasculares, posiblemente relacionado con el escaso número de pacientes, el buen control metabólico y la insulinoterapia intensificada desde el diagnóstico. En una cohorte de pacientes con DM1 del estudio epidemiológico Pittsburg se encontró que la TeDG fue mejor predictor de enfermedad coronaria que el control glucémico $(\mathrm{HbA} 1 \mathrm{c})^{31}$. En el estudio Diabetes Control and Complications Trial (DCCT) se indicó que los pacientes con resistencia a la insulina evaluados por una baja TeDG presentaron mayor riesgo de desarrollar complicaciones microvasculares y macrovasculares, independientemente del grupo de tratamiento asignado ${ }^{32}$.

La IR es un factor de riesgo para el desarrollo de nefropatía diabética en adultos con DM1. En nuestro estudio, el análisis multivariado reveló que los pacientes que se encontraban en el tercil más bajo de TeDG tuvieron tres veces más riesgo de nefropatía diabética. Datos similares se publicaron en el estudio FinnDiane ${ }^{33}$. La asociación entre la sensibilidad a la insulina y la nefropatía diabética no es un hallazgo reciente. En 1993 Yip et al. evaluaron 14 pacientes con DM1 y observaron que aquellos con microalbuminuria tuvieron una TeDG significativamente más baja durante el clamp euglucémico-hiperinsulinémico, incluso después de ajustar por presión arterial e IMC ${ }^{34}$. Más tarde, Orchard et al. en el año 2002 encontraron resultados similares ${ }^{35}$. En 2015, Bjornstad et al. descubrieron que los pacientes con DM1 y albuminuria que regresaron a normoalbuminuria después de seis años de seguimiento presentaban más insulinosensibilidad que aquellos con albuminuria persistente ${ }^{36}$.

Esto pone en evidencia que la insulinorresistencia y los factores asociados a ella como la hipertensión arterial, la dislipemia, el mal control metabólico y los factores proinflamatorios "aceleran" el desarrollo de complicaciones en estos pacientes.

No se ha establecido un umbral específico de TeDG para definir la presencia de IR en DM1. En el estudio de Chillarón las complicaciones de la diabetes ocurrieron exclusivamente en pacientes en el tercil más bajo de TeDG utilizando como punto de corte $8,16^{10}$. Olson et al. demostraron que pertenecer al quintil inferior de TeDG $(<6,22)$ fue un predictor independiente de mortalidad global en el estudio epidemiológico de Pittsburgh ${ }^{37}$. En nuestro trabajo los pacientes en el tercil inferior $(<6,54)$ manifestaron asociación independiente con nefropatía y cualquier complicación relacionada con la diabetes.

Nuestro estudio presenta como limitaciones el diseño retrospectivo, por lo cual no puede establecerse una relación de causalidad entre una menor TeDG y la presencia de complicaciones micro y macrovasculares. Además los pacientes que se incluyeron en el estudio tenían diagnóstico clínico de DM1 con una proporción baja de medición de autoanticuerpos específicos (ac anti-GAD, ac IA2A, ZnT8) que respaldaran el diagnóstico.

\section{CONCLUSIONES}

El fenotipo de los pacientes con DM1 está cambiando en relación a los hábitos poco saludables como la mala alimentación y el sedentarismo. Además la insulinoterapia intensificada para obtener los objetivos terapéuticos acentúa esta situación a través del incremento de peso. En la práctica clínica, la determinación de la TeDG podría identificar pacientes con mayor insulinorresistencia a fin de implementar estrategias más agresivas para el manejo de los factores de riesgo. Se necesitan trabajos prospectivos y aleatorizados para valorar la eficacia y seguridad de intervenciones que mejoren la insulinosensibilidad como la dieta, el ejercicio y el uso de insulinosensibilizadores. 


\section{BIBLIOGRAFÍA}

1. Ginsberg HN. Investigation of insulin sensitivity in treated subjects with ketosis-prone diabetes mellitus. Diabetes 1977; 26: 278-283.

2. DeFronzo RA, Simonson D, Ferrannini E. Hepatic and peripheral insulin resistance: a common feature of type 2 (non-insulindependent) and type 1 (insulin-dependent) diabetes mellitus. Diabetologia 1982; 23:313-319.

3. DeFronzo RA, Hendler R, Simonson D. Insulin resistance is a prominent feature of insulin-dependent diabetes. Diabetes 1982; 31:795-801.

4. Chillarón JJ, Flores-Le-Roux JA, Goday A, et al. Metabolic syndrome and type- 1 diabetes mellitus: prevalence and associated factors. Rev Esp Cardiol 2010; 63:423- 429

5. Thorn LM, Forsblom C, Fagerudd J, et al; FinnDiane Study Group. Metabolic syndrome in type 1 diabetes: association with diabetic nephropathy and glycemic control (the FinnDiane Study). Diabetes Care 2005; 28:2019-2024.

6. Rodrigues TC, Canani LH, Gross JL. Metabolic syndrome, insulinresistance and cardiovascular disease in type-1 diabetes mellitus. Arq Bras Cardiol 2010;94: 134-139.

7. Libman IM, Becker DJ. Coexistence of type 1 and type 2 diabetes mellitus: "double" diabetes? Pediatr Diabetes 2003; 4:110-113.

8. Gilliam LK, Brooks-Worrell BM, Palmer JP, et al. Autoimmunity and clinical course in children with type 1, type 2, and type 1.5 diabetes. J Autoimmun 2005; 25:244-250.

9. Williams KV, Erbey JR, Becker D, et al. Can clinical factors estimate insulin resistance in type 1 diabetes? Diabetes 2000; 49:626-632.

10. Chillarón JJ, Goday A, Flores-Le-Roux JA, et al. Estimated glucose disposal rate in assessment of the metabolic syndrome and microvascular complications in patients with type 1 diabetes. J Clin Endocrinol Metab 2009; 94:3530-3534.

11. Epstein EJ, Osman JL. Use of the estimated glucose disposal rate as a measure of insulin resistance in an urban multiethnic population with type 1 diabetes. Diabetes Care 2013; 36: 2280-2285.

12. Tercera Encuesta Nacional de Factores de Riesgo para Enfermedades No Transmisibles. Disponible en: www. indec.gov.ar/bajarPublicacion. asp?idc=3CA3D716DAC D1FEED191A1BE551949F175343FDAFF106A623D694B04340DC484EA3967340FD9212F. Argentina 2013. www. msal.gob.ar/images/stories/bes/graficos/0000000544c nt-2015_09_04_encuesta_nacional_factores_riesgo.pdf

13. Levey AS, Bosch JP. A more accurate method to estimate glomerular filtration rate from serum creatinine: a new prediction equation. Modification of diet in renal disease Study Group. Ann Intern Med 1999 Mar 16; 130(6):461-70.

14. American Diabetes Association. Standards of Medical Care in Diabetes. Diabetes Care 2014 Jan; 37 (Supplement 1): S14-S80.
15. Office of Disease Prevention and Health Promotion, U.S. Department of Health and Human Services 2014. Disponible en: www.health.gov/paguidelines/guidelines/default.aspx.

16. Alberti K, Zimmet P. The metabolic syndrome a new world wide definition. Lancet 2005; 366 (9491):10591062.

17. Diabetic retinopathy: a position statement by the American Diabetes Association. Diabetes Care 2017; 40:412418.

18. American Diabetes Association. Consensus development conference on the diagnosis and management of nephropathy in patients with diabetes mellitus. Diabetes Care 1994; 17:1357.

19. Dyck PJ. Approches to improve epidemiological studies of diabetic neuropathy. Diabetes 1997; S5-S13.

20. The DCCT Research Group. Weight gain associated with intensive therapy in the diabetes control and complications trial. Diabetes Care 1988, 11:567-573.

21. Libman IM, Pietropaolo M. Changing prevalence of overweight children and adolescents at onset of insulintreated diabetes. Diabetes Care 2003, 26:2871-2875.

22. Minges KE, Whittemore R, Grey M. Overweight and obesity in youth with type 1 diabetes. Annu Rev Nurs Res 2013; 31:47-69.

23. Chillarón JJ, Flores Le-Roux JA. Type 1 diabetes, metabolic syndrome and cardiovascular risk. Metabolism 2014; 63:181-187.

24. Leroux C, Brazeau AS, Gingras V, et al. Lifestyle and cardiometabolic risk in adults with type 1 diabetes: a review. Can J Diabetes 2014; 38:62-69.

25. Merger SR, Leslie RD. The broad clinical phenotype of type 1 diabetes at presentation. Diabet Med 2013; 30:170-8.

26. Merger SR, Kerner W. Prevalence and comorbidities of double diabetes. Diabetes research and clinical practice 2016; 119, 48-56.

27. Intensive Diabetes Management. Implications of the DCCT and UKPDS. Diabetes Educ 2002; 28:735-40.

28. Thorn L, Forsblom C. Effect of parental type 2 diabetes on offspring with type 1 diabetes. Diabetes Care 2009; 32:63-68.

29. Martin $\mathrm{FI}$, Stocks AE. Insulin sensitivity and vascular disease in insulin-dependent diabetics. Br Med J 1968; 2:81-82.

30. Chillarón JJ, Flores-Le-Roux JA. Síndrome metabólico y diabetes mellitus tipo 1: prevalencia y factores relacionados. Rev Esp Cardiol 2010; 63(4):423-9.

31. OrchardTJ, Olson JC. Insulin resistance related factors, but not glycemia, predict coronary artery disease in type 1 diabetes: 10- year follow-up data from the Pittsburgh Epidemiology of Diabetes Complications Study. Diabetes Care 2003; 26:1374-1379. 
32. Kilpatrick E, Rigby A, et al. Insulin resistance, the metabolic syndrome, and complication risk in type 1 diabetes "double diabetes" in the Diabetes Control and Complications Trial. Diabetes Care 2007; 30:707-712.

33. Thorn LM, Forsblom C. The FinnDiane Study Group: metabolic syndrome in type 1 diabetes: association with diabetic nephropathy and glycemic control (the FinnDiane study). Diabetes Care 2005; 28:2019-2024.

34. Yip J, Matlock MB. Insulin resistance in insulin-dependent diabetic patients with microalbuminuria. Lancet 1993; 342:883-887
35. Orchard T, Chang Y, Ferrell R, et al. Nephropathy in type 1 diabetes: a manifestation of insulin resistance and multiple genetic susceptibilities? Further evidence from the Pittsburgh Epidemiology of Diabetes Complication Study. Kidney International 2002; Vol. 62, 963970.

36. Bjornstad $P$, Maahs M. Estimated insulin sensitivity predicts regression of albuminuria in type 1 diabetes. Diabet Med 2015 Fe; 32(2): 257-261.

37. Olson JC, Erbey JR, Williams KV. Subclinical atherosclerosis and estimated glucose disposal rate as predictors of mortality in type 1 diabetes. Ann Epidemiol 2002; 12:331-337. 